\title{
Diagnosis on Welding Defect Signals Using Sample Entropy
}

\author{
Gao Yatian ${ }^{1}$, Leng Jiancheng ${ }^{*}, 2$, Xu Mingxiu ${ }^{3}$ and Xing Haiyan ${ }^{2}$
}

${ }^{1}$ School of Computer \& Information Technology, Northeast Petroleum University, Daqing, Heilongjiang, 163318, P.R. China; ${ }^{2}$ School of Mechanical Science and Engineering, Northeast Petroleum University, Daqing, Heilongjiang, 163318, P.R. China; ${ }^{3}$ School of Mathematics and Physics, University of Science and Technology Beijing, Beijing, 100083, P.R. China

\begin{abstract}
A new diagnostic method to identify alternating current field measurement (ACFM) signal based on sample entropy combined with wavelet packet feature is put forward in order to accurately evaluate the damage degree of welding defects. A butt welded tubular specimen with three kinds of different welding qualities was inspected and the corresponding ACFM signals were recorded by a commercial instrument. Subsequently sample entropies of the original signals and their wavelet package coefficients were computed respectively and compared via the bar graphs. The results show that the sample entropy successfully discriminates between different welding defects, and moreover it can be utilized to detect early or slight damage, demonstrating that the proposed approach is a promising and effective tool in characterizing the ACFM signals of welding defects.
\end{abstract}

Keywords: Alternating current field measurement, sample entropy, wavelet package, welding defect.

\section{INTRODUCTION}

Welding is being widely used for construction of various engineering structures in petroleum industry, and inevitably some flaws will occur during welding and long-term service. For this purpose, a new electromagnetic nondestructive testing technique, named alternating current field measurement (ACFM) has been pioneered to detect surface and subsurface flaws, especially crack defects and underwater welding quality [1], and has attracted much attention because it can estimate both defect length and depth simultaneously by measuring two magnetic field components $\mathrm{Bx}$ and $\mathrm{Bz}$ without calibration. Raine et al. summarized the principles, developments of the technique and its applications in detail [2], and $\mathrm{Li}$ et al. investigated the rules and characteristics of the electromagnetic signal distribution in the defected area using finite element simulation [3]. Also, a wavelet network scheme was proposed for inverting ACFM crack signals to the depth profile of a surface crack with no geometrical shape [4]. So far, the ACFM technique has been widely applied in many fields, such as offshore platforms [5], highspeed inspection of rails [6], drill collar threaded connections [7], etc.

However, the ACFM signal is nonlinear and nonstationary, susceptible to noise interference, such as scanning speed, fluctuation and lift-off value of probes, etc. The approximate entropy (ApEn) was first developed to measure the complexity of time series by Pincus in 1990 [8], and can be applied to deterministic and stochastic systems compared to the parameters of nonlinear dynamics such as correlation dimension, Lyapunov exponent. However, ApEn is biased

*Address correspondence to this author at the School of Mechanical Science and Engineering, Northeast Petroleum University, Daqing, Heilongjiang, 163318, P.R. China; Tel/Fax: +86 459 6503884; E-mail: lbyljc@163.com and even leads to inconsistent results due to self-matches. More recently, Richman et al. improved the ApEn method and introduced the sample entropy (SampEn) to quantify the irregularity of a sequence of numbers [9], which has been successfully utilized to estimate physiological data [10], electromagnetically acoustic emission signals [11], and bearing fault diagnosis [12], etc. owing to less sensitivity to the length of data.

The objective of the present work is to analyze quantitatively the damage degree of welding defects by virtue of SampEn and wavelet package. In this respect, a satisfactory estimation could allow the prediction time series regularity, thus identifying the early and serious damage successfully.

\section{INTRODUCTION OF SAMPLE ENTROPY}

SampEn can be computed as follows formally, given a standardized time series $u_{1}, u_{2}, \cdots, u_{N}$ with $N$ data points [11]:

The first step of the algorithm is to define vector sequences of length $m$ given by

$$
x(i)=[u(i), u(i+1), \cdots, u(i+m-1)], i=1,2, \cdots, N-m+1
$$

And then define the distance $d[x(i), x(j)]$ between vectors $\left\{x_{i}\right\}$ and $\left\{x_{j}\right\}$, as the absolute maximum difference between them, i.e.

$$
d[x(i), x(j)]=\max _{k=0, \cdots, m-1}|u(i+k)-u(j+k)|
$$

Subsequently, the following quantity is defined for a given tolerance $r$

$$
B_{i}^{m}(r)=\frac{\operatorname{num}\{d[x(i), x(j)] \leq r\}}{N-m-1}, 1 \leq i \leq N-m(j \neq i)
$$

Thus the density is calculated as follows 
$B^{m}(r)=\frac{\sum_{i=1}^{N-m} B_{i}^{m}(r)}{N-m}$

The next step of the process is to increase the dimension to $m+1$ and calculate $B^{m+1}(r)$

$$
B^{m+1}(r)=\frac{\sum_{i=1}^{N-m} B_{i}^{m+1}(r)}{N-m}
$$

Finally, SampEn is estimated by the following formula

$$
\operatorname{SampEn}(m, r, N)=-\ln \frac{B^{m+1}(r)}{B^{m}(r)}
$$

It needs to be noted that the above input parameters $m$ and $r$ must be selected to computer SampEn, where $m$ is the length of sequences to be compared and $r$ is the tolerance for receiving matches. In the present study, $M$ is set by 2 and $r$ $0.2 \times \mathrm{SD}$, where $\mathrm{SD}$ is the standard deviation of the original time series. Since SampEn avoids counting self-matches and is independent of the record length, it is more consistent than ApEn.

\section{EXPERIMENTAL}

The experimental data were obtained from a butt welded tubular specimen with artificial defects including a slag inclusion and a crack defect as shown in Fig. (1), using the ACFM method. The specimen was inspected along the weld toes by a commercial TSC's AMIGO crack microgauge and a single channel standard weld probe operated at a frequency of $5 \mathrm{kHz}$, as shown in Fig. (2).

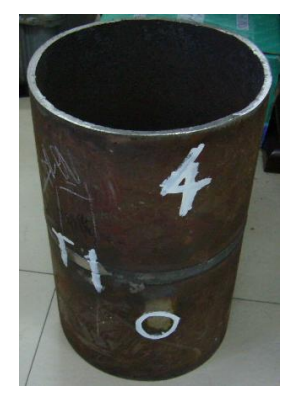

Fig. (1). Butt welded tubular specimen.

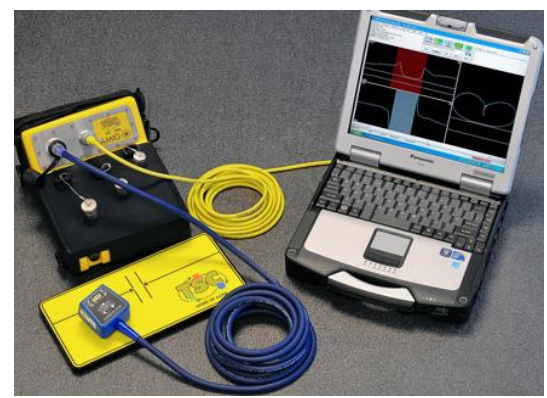

Fig. (2). ACFM measurement instrument.

The ACFM signals corresponding to different welding defects including good quality, slag inclusion and crack defect were recorded in received condition without any clean- ing by the above ACFM measurement system, respectively. Note that the sensor probe was always moved as smoothly and uniformly as possible at a velocity of $10-25 \mathrm{~mm} / \mathrm{s}$.

\section{ACFM SIGNAL ANALYSIS BASED ON SAMPLE ENTROPY}

The original ACFM signals detected were displayed in Figs. (3-5), from upper to lower graph corresponding to good welding quality, slag inclusion and crack defect, respectively.
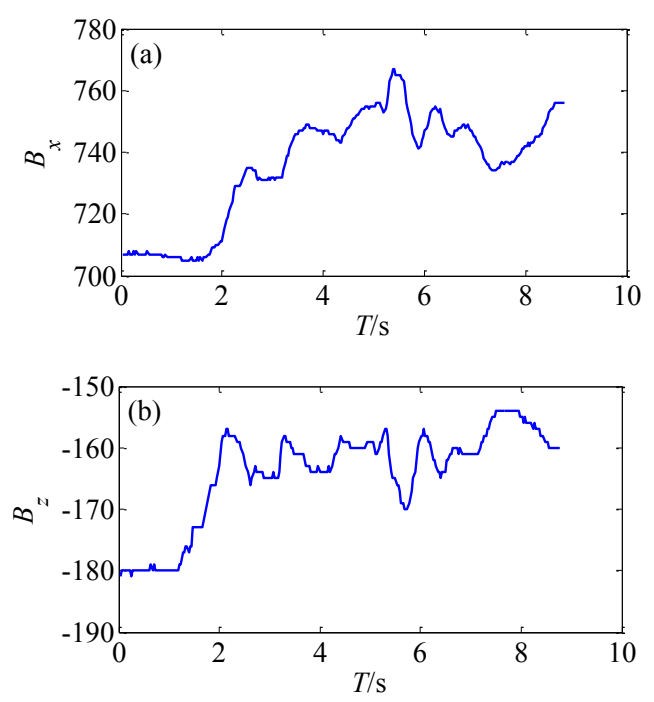

Fig. (3). Original ACFM signals corresponding to good welding quality.
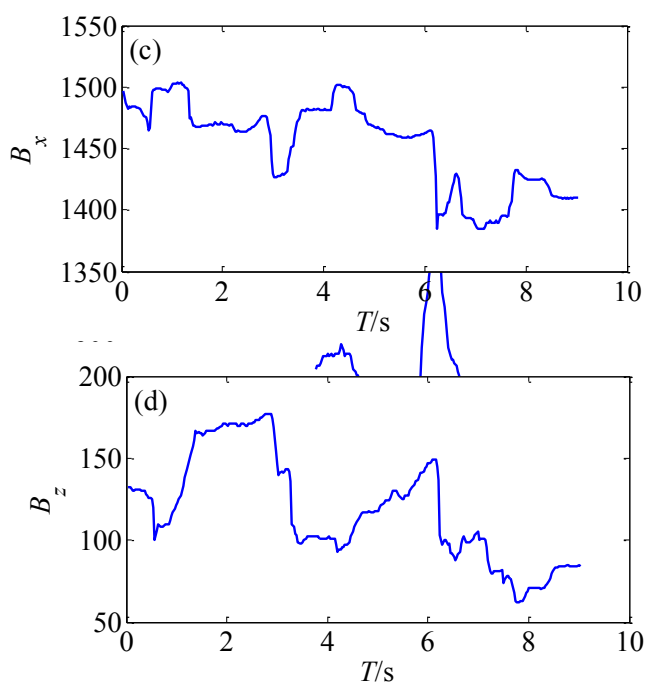

Fig. (4). Original ACFM signals corresponding to slag inclusion.

It can be seen clearly from Figs. (3-5) that the lower four curves fluctuated more obviously, especially Fig. (5) reveals a recognizable signal feature corresponding to crack defect, with Bx exhibiting a minimum in field strength and $\mathrm{Bz}$ exhibiting a wave crest and trough. Nevertheless, it is still difficult to distinguish the three types of welding defects among good quality, slag inclusion and crack defect only by virtue of different wave forms. 
In order to further analyze and identify different welding signals, the wavelet package-based sample entropy method was utilized to measure the complexity of signals quantitatively. Figs. (6-8) give the wavelet package coefficient distributions corresponding to the signals in Figs. (3-5), respectively using db4 orthogonal wavelet package.
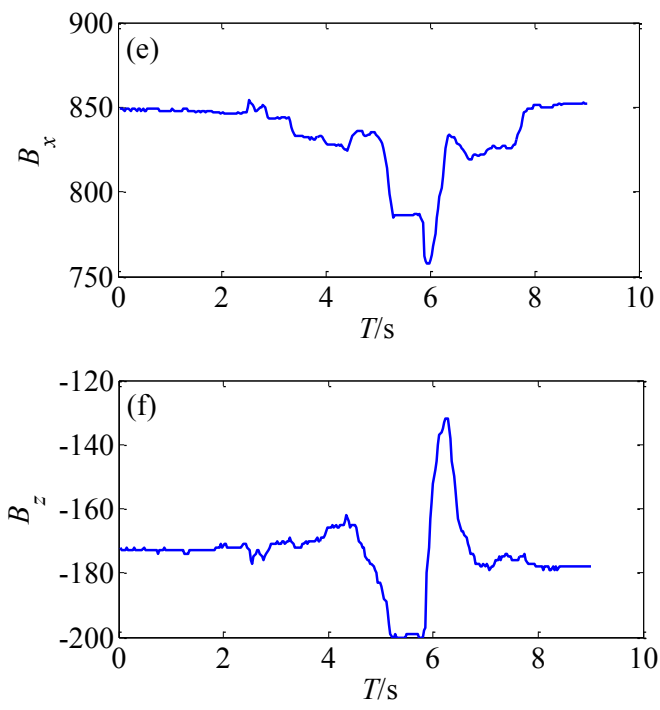

Fig. (5). Original ACFM signals corresponding to crack defect.
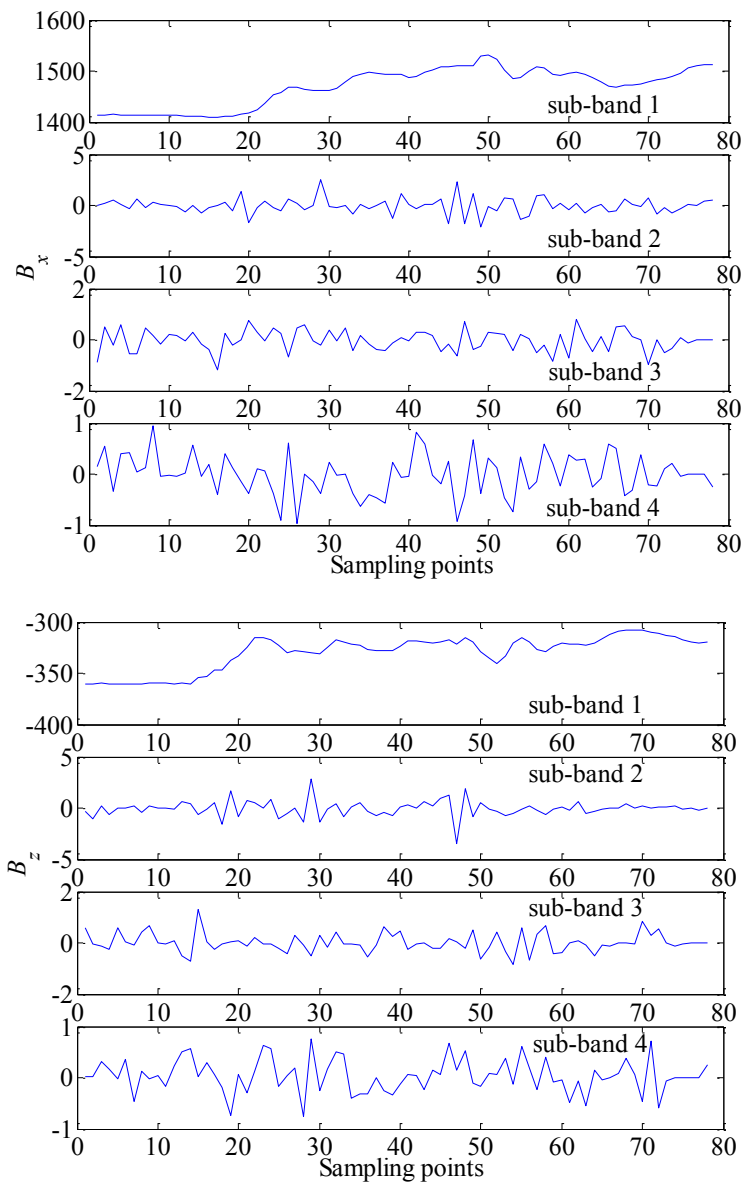

Fig. (6). Wavelet packet coefficient distribution of the good welding signal.
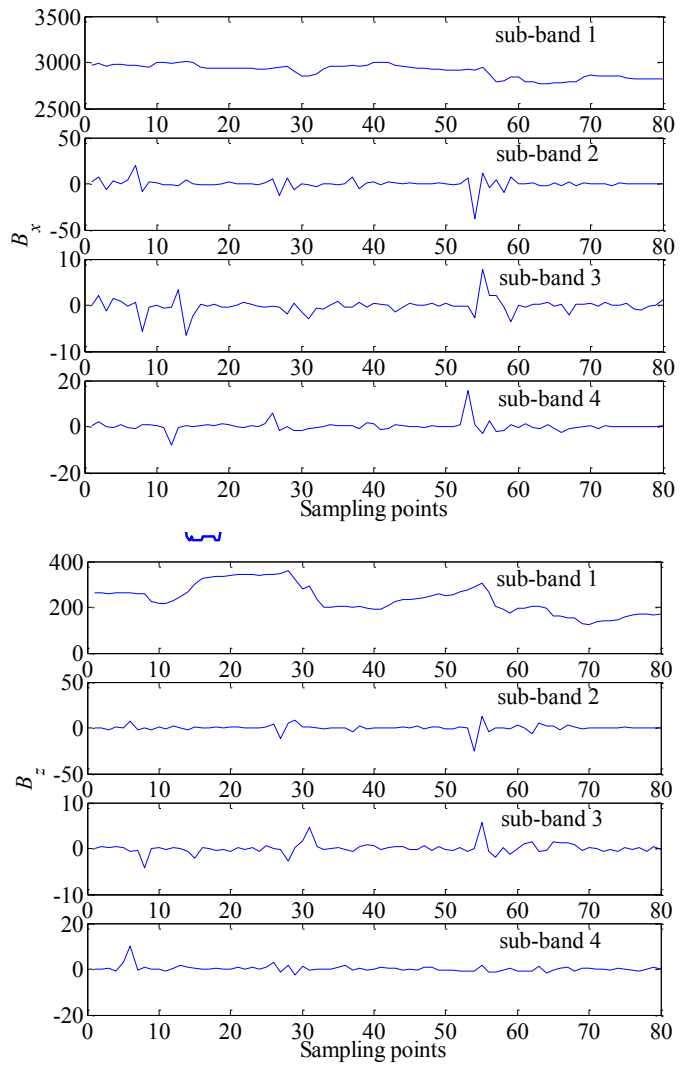

Fig. (7). Wavelet packet coefficient distribution of the slag inclusion signal.
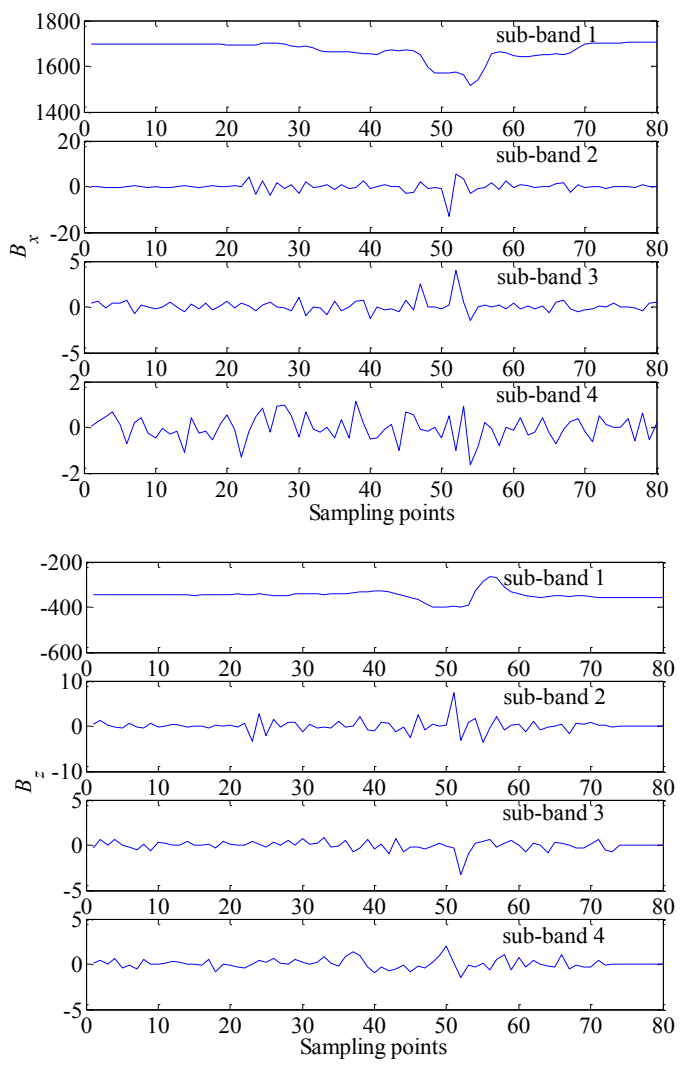

Fig. (8). Wavelet packet coefficient distribution of the crack defect signals. 
Table 1. Sample entropy of different defect signals in each sub frequency band.

(1-1)

\begin{tabular}{|c|c|c|c|c|}
\hline \multicolumn{2}{|c|}{ Signal } & Sample entropy of the original & $\begin{array}{c}\text { Sample entropy of the frequencies sub-bands decomposed by } \\
\text { wavelet package }\end{array}$ \\
\cline { 3 - 5 } & & signal & 1 & $\mathbf{2}$ \\
\hline \hline \multirow{3}{*}{ Good welding quality } & Bx signal & 1.7325 & 1.7214 & 1.6839 \\
\cline { 2 - 5 } & Bz signal & 1.7316 & 1.6956 & 1.6908 \\
\hline \multirow{3}{*}{ Slag inclusion } & Bx signal & 2.0503 & 1.9885 & 1.9124 \\
\cline { 2 - 5 } & Bz signal & 2.1070 & 2.0795 & 1.9457 \\
\hline \multirow{3}{*}{ Crack defect } & Bx signal & 1.3017 & 1.1727 & 1.0800 \\
\cline { 2 - 5 } & Bz signal & 1.2989 & 1.0901 & \multirow{2}{*}{0.9383} \\
\hline
\end{tabular}

(1-2)

\begin{tabular}{|c|c|c|c|}
\hline \multicolumn{2}{|c|}{ Signal } & \multicolumn{2}{c|}{ Sample entropy of the frequencies sub-bands decomposed by wavelet package } \\
\cline { 3 - 4 } & & $\mathbf{3}$ & $\mathbf{4}$ \\
\hline \hline \multirow{3}{*}{ Good welding quality } & Bx signal & 1.5843 & 1.5638 \\
\cline { 2 - 4 } & Bz signal & 1.6849 & 1.5858 \\
\hline \multirow{2}{*}{ Slag inclusion } & Bx signal & 1.8210 & 1.7523 \\
\cline { 2 - 4 } & Bz signal & 1.8122 & 1.7960 \\
\hline \multirow{2}{*}{ Crack defect } & Bx signal & 0.8855 & 0.6919 \\
\cline { 2 - 4 } & Bz signal & 0.8161 & 0.6315 \\
\hline
\end{tabular}
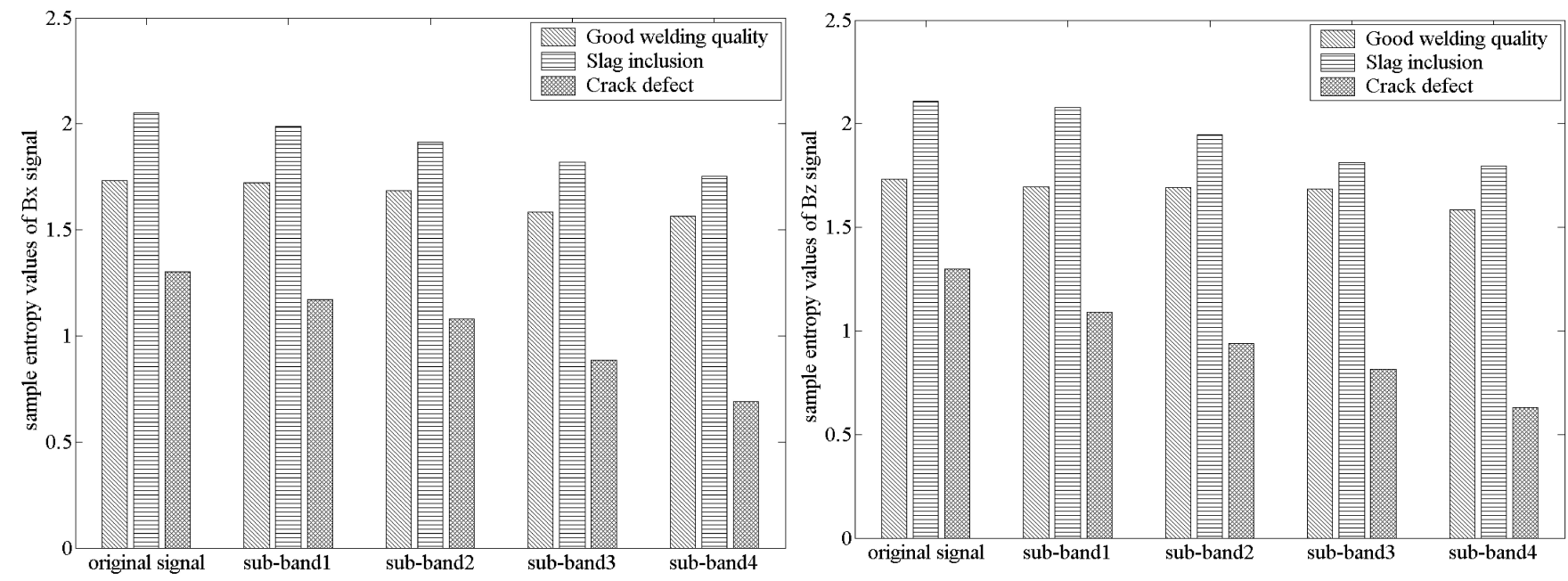

Fig. (9). Comparison of sample entropy among different signals.

Accordingly, the parameters are set as follows: $m=2$ and $r=0.2$, and the sample entropy values are calculated as listed in Table 1.

It follows from Table $\mathbf{1}$ that the sample entropy values are different under three different damage states, however, they do not increase with increasing damage degree. It is interesting that the sample entropy value of the slag inclusion signal is the largest whilst that of the crack defect signal is the smallest, i.e., the representative signal response from a crack defect is less complex than other two types of signals, which is in agreement with the research results in fault diagnosis [13].

The bar graphs corresponding to the data in Table $\mathbf{1}$ are constructed in Fig. (9) in order to better represent the relationship among the sample entropy of different signals. Since the signal with slag inclusion is more complex than that with good welding quality, the former sample entropy value exhibits larger. On the other hand, the $\mathrm{Bx}$ and $\mathrm{Bz}$ sig- 
nals with crack defect display typical abnormal wave characteristics respectively, thus the corresponding sample entropy values decrease obviously. It follows from Fig. (9) that the sample entropy values of the original signal and each sub frequency band corresponding to different welding qualities both can be used to characterize the complexity degree of signal serials quantitatively, and also the sample entropy is sensitive to the slight damage such as slag inclusion, indicating that the sample entropy has potentials in early diagnosis of welding defects.

\section{CONCLUSION}

This work proposes a novel application of sample entropy combined with wavelet package as a quantitative method to evaluate the welding defects. Three kinds of signals representing different welding qualities are first measured using ACFM technique, and the corresponding wavelet package coefficient distributions are obtained, Through the SampEn algorithm, the results show that the SampEn value of the slag inclusion signal increases markedly as compared to the signal with good welding quality, whilst it becomes minimum for the crack defect signal, demonstrating that the sample entropy methodology is capable to characterize complexity features of ACFM defect signals in terms of their irregularity. Furthermore, the sample entropy is sensitive to the early damage, which could be a good dynamical signature to distinguish different damage degrees, providing a relatively simple approach to evaluate welding defects quantitatively.

\section{CONFLICT OF INTEREST}

The authors confirm that this article content has no conflict of interest.

\section{ACKNOWLEDGEMENTS}

This work was financially supported by Scientific Research Fund of Heilongjiang Provincial Education Department (12541100), National Natural Science Foundation of China (11472076 and 11272084), China Postdoctoral Science Foundation (2013M541337), and Heilongjiang Postdoctoral Foundation of China (LBH-Z13050).

\section{REFERENCES}

[1] M.C. Lugg, R. Collins, and D.R. Parramore, "Alternating current field measurement technique: recent work related to the aerospace industry", In: Proceedings of the $20^{\text {th }}$ Annual British Conference on Non-Destructive Testing, 1986, pp. 535-549.

[2] A. Raine, and M. Lugg, "A review of the alternating current field measurement inspection technique", Sensor Review, vol. 19, pp. 207-213, 1999.

[3] W. Li, G.M. Chen, C.R. Zhang, and T. Liu, "Simulation analysis and experimental study of defect detection underwater by ACFM probe", China Ocean Engineering, vol. 27, pp. 277-282, 2013.

[4] M. Ravan, S.H.H. Sadeghi, and R. Moini, "Using a wavelet network for reconstruction of fatigue crack depth profile from $\mathrm{AC}$ field measurement signals", NDT \& E International, vol. 40, pp. 537-544, 2007.

[5] P. Chakrabarti, I. Abu-Odeh, A. Mukkamala, B. Majumdar, M.H. Faber, D. Straub, and J.O. Ramirez, "An overview of the reassessment studies of fixed offshore platforms in the bay of Campeche, Mexico", ASME $24^{\text {th }}$ International Conference on Offshore Mechanics and Arctic Engineering, vol. 1, pp. 121-134, 2005.

[6] M.Ph. Papaelias, M.C. Lugg, C. Roberts, and C.L. Davis, "Highspeed inspection of rails using ACFM techniques", NDT \& E International, vol. 42, pp. 328-335, 2009.

[7] M.J. Knight, F.P. Brennan, and W.D. Dover, "Effect of residual stress on ACFM crack measurements in drill collar threaded connections”, NDT \& E International, vol. 37, pp. 337-343, 2004.

[8] S.M. Princus, "Approximate entropy as a measure of system complexity", Proceedings of the National Academy of Sciences of the United States of America, vol. 88, pp. 2297-2301, 1991.

[9] J.S. Richman, and J.R. Moorman, "Physiological time-series analysis using approximate entropy and sample entropy", America Journal of Physiology Heart and Circulatory Physiology, vol. 278, pp. 2039-2049, 2000.

[10] W.T. Chen, J. Zhuang, W.X. Yu, and Z.Z. Wang, "Measuring complexity using FuzzyEn, ApEn, and SampEn", Medical Engineering \& Physics, vol. 31, pp. 61-68, 2009.

[11] S.Z. Liu, J. Li, C. Zhang, X.Q. Shen, L. Jin, and Q.X. Yang, "Research on electromagnetically acoustic emission signals using sample entropy", In: 6th International Conference on Electromagnetic Field Problems and Applications, 2012, pp. 1-4.

[12] L. Zhang, G.L. Xiong, H.S. Liu, H.J Zou, and W.Z. Guo, "Bearing fault diagnosis using multi-scale entropy and adaptive neuro-fuzzy inference", Expert Systems with Applications, vol. 37, pp. 60776085, 2010.

[13] Z.H. Zhao, and S.P. Yang, "Sample entropy-based roller bearing fault diagnosis method", Journal of Vibration and Shock, vol. 31, pp. 136-140, 2012. (In Chinese)

\author{
Received: May 26, 2015 \\ Revised: July 14, 2015 \\ Accepted: August 10, 2015 \\ (C) Yatian et al.; Licensee Bentham Open.
}

This is an open access article licensed under the terms of the (https://creativecommons.org/licenses/by/4.0/legalcode), which permits unrestricted, noncommercial use, distribution and reproduction in any medium, provided the work is properly cited. 